\title{
Electrodeposited PdNi as possible ferromagnetic contacts for Carbon nanotubes.
}

\author{
A. R. Usgaocar ${ }^{\star}, 1$, C.H. de Groot $^{1}$ \\ ${ }^{1}$ Nano Research Group, School of Electronics and Computer Science, University of Southampton, Southampton SO17 1BJ, UK
}

Received XXXX, revised XXXX, accepted XXXX

Published online XXXX

PACS 73.61.-r; 73.63.-b; 75.70.-i; 81.07.-b; 82.45.-h; 85.35.Kt

* Corresponding author: e-mail aru07r@ecs.soton.ac.uk, Phone: +44-23-8059-2422, Fax: +44-23-8059-3029

A process for electrodepositing PdNi alloys and subsequent characterisation studies are reported. PdNi alloys are deposited on 0.019-0.021 $\Omega . \mathrm{cm}$ and 1-2 $\Omega . \mathrm{cm} \mathrm{n}$ type Silicon from a bath of Pd-ethylenediamine dichloride and Ni sulphate. The deposited films form excellent Schottky barriers on 1-2 $\Omega$.cm Si with leakage currents of the order of $\mu \mathrm{A} / \mathrm{cm}^{2}$ and forward current higher than the reverse current by about six orders of magnitude at $1 \mathrm{~V}$. Ni atomic fractions in the deposited films are studied for different bath concentrations and the deposition potential was found to play an important role in deciding the composition of the deposited film. For high Ni concentration solutions, it is possible to deposit films with a wide range of $\mathrm{Ni}$ content by varying the deposition potential, while this method cannot be used with low $\mathrm{Ni}$ concentration solutions. Films with Ni concentrations above $30 \%$ were observed to be ferromagnetic at room temperature and ferromagnetic properties strengthened with $\mathrm{Ni}$ content. A structure for a carbon nanotube device is proposed and electrical characterisations of the device using PdNi alloy contacts are presented. The Schottky barrier characteristics and ferromagnetic character make PdNi alloys a good material for ferromagnetic contacts to CNTs on a Si substrate.

Copyright line will be provided by the publisher

1 Introduction Carbon nanotubes (CNTs) are being actively researched as a replacement for $\mathrm{Si}$ in future electronic devices due to their attractive electronic and thermal properties [1,2]. Research effort has been focussed on replacing the Si channel with a CNT and using MOSFET operating principles $[3,4]$ and on exploiting new phenomena like spin based electronics [5,6]. It emerges from these studies that the metal-CNT contact is extremely important in determining the characteristics of the device. Javey et al. [3] have shown that Pd forms good contacts with CNTs. Sahoo et al. [5] used evaporated PdNi alloys to examine some spin based phenomena in CNTs while Tombros et al. [6] have demonstrated complete decoupling of spin and charge currents in a CNT.

Kiziroglou et al. [7] have observed a dramatic improvement in the reverse bias characteristics of electrodeposited Ni-Si Schottky barriers compared to evaporated barriers. In this research, we investigate whether a similar improvement in metal-CNT contact quality can be ob- served, thereby improving spintronic devices that use evaporated PdNi as contacts.

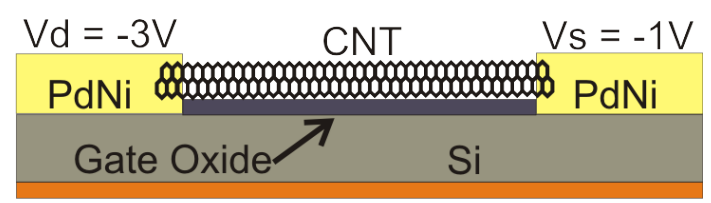

Aluminium Backcontact (OV)

Figure 1 Fabricated structure and biasing scheme of the device.

The structure of the CNT spin transistor is shown in Fig. 1. The operation of this device as a transistor relies on the drain-gate and source-gate schottky barriers having low leakage currents when reverse biased, so as to maximise the current through the CNT. The biasing scheme of Fig. 1 reverse biases both Schottky barriers while maintaining a potential across the carbon nanotube. The electrical characterisation of the reverse biased Schottky barriers is 
important to ensure that the device can be used as intended for future spintronic applications.

The magnetic characterisation of electrodeposited PdNi alloys is also essential to determine their suitability as ferromagnetic contacts. In the following sections the electrodeposition, electrical characterisation and magnetic characterisation of the PdNi films on Si are described.

2 Electrodeposition The recipe for the electrochemical bath solution was taken from Ref. [8] and used Nisulphate, Pd-ethylenediamine dichloride, Ammonium sulphate and Ammonia with deionised(DI) water as the solvent. Different solutions were prepared by varying the $\mathrm{NiSO}_{4}$ concentration from $16.65 \mathrm{~g} / \mathrm{L}$ to $49.95 \mathrm{~g} / \mathrm{L}$ and the $\mathrm{Pd}($ en $) \mathrm{Cl}_{2}$ concentration from $38.85 \mathrm{~g} / \mathrm{L}$ to $5.55 \mathrm{~g} / \mathrm{L}$ such that the numerical total of the two was $55.5 \mathrm{~g} / \mathrm{L}$. The concentration of Ammonium Sulphate was kept constant. If required, the $\mathrm{pH}$ was adjusted to 7-7.5 using $\mathrm{H}_{2} \mathrm{SO}_{4}$ and Ammonia, but the additional volume of Ammonia used was at most $0.5 \mathrm{ml} / \mathrm{L}$. Three solutions were prepared for each combination of $\mathrm{Ni}$ and $\mathrm{Pd}$ salts as shown in Table. 1.

Table 1 Concentrations of components in the three solutions prepared for each combination of $\mathrm{Ni}$ and $\mathrm{Pd}$ salts.

\begin{tabular}{lccc}
\hline Components & Ni bath $(\mathrm{g} / \mathrm{L})$ & Pd bath $(\mathrm{g} / \mathrm{L})$ & PdNi bath $(\mathrm{g} / \mathrm{L})$ \\
\hline $\mathrm{NiSO}_{4} .6 \mathrm{H}_{2} \mathrm{O}$ & 44.40 & 0 & 44.40 \\
$\mathrm{Pd}(\mathrm{en}) \mathrm{Cl}_{2}$ & 0 & 11.1 & 11.1 \\
$\left(\mathrm{NH}_{4}\right)_{2} \mathrm{SO}_{4}$ & 16.70 & 16.70 & 16.70 \\
$\mathrm{NH}_{3}(35 \%)$ & $45 \mathrm{ml} / \mathrm{L}$ & $45 \mathrm{ml} / \mathrm{L}$ & $45 \mathrm{ml} / \mathrm{L}$ \\
\hline
\end{tabular}

Chips of size $1.5 \mathrm{~cm} \times 1 \mathrm{~cm}$ were cleaved from an $\mathrm{n}$ type, $<100>\mathrm{Si}$ wafer of resistivity 0.019-0.021 $\Omega$.cm. The chips were washed with Acetone and Isopropylalcohol (IPA), blown dry and coated with an electrically inert varnish to prevent electrodeposition in all areas except a $1 \mathrm{~cm} \times 0.5 \mathrm{~cm}$ window. The native oxide was etched using 20:1 HF followed by washing with DI water and electrodeposition was performed at room temperature using an Autolab PGSTAT30 electrochemical system with a calomel reference electrode and a Pt mesh as the counter electrode. The devices formed were used to study the composition and magnetic properties of the films.

A second set of chips from an n-type $<100>$ Si wafer of resistivity $1-2 \Omega$.cm with ohmic Al backcontacts were used to study the electrical characteristics of the PdNi-Si Schottky barriers. A $20 \mathrm{~nm}$ thermal oxide was grown on the front side of the chips and patterned to form sets of circular and square contacts with sizes ranging from $1.5 \mathrm{~mm}$ $0.2 \mathrm{~mm}$. The thermal oxide was used as an insulating layer to restrict metal deposition to only the active area of the chip.

Cyclic voltammetry curves were measured for each solution by sweeping the electrode potential from 0 to $-3 \mathrm{~V}$ and back while measuring the current in the electrochemical cell. A high potential pulse of $-2.5 \mathrm{~V}$ was applied for
$0.1 \mathrm{~s}$ to promote nucleation of the metal on the Si surface and increase the smoothness of the deposit [7]. PdNi films were deposited at a constant potential chosen such that the current density was in the $1-3 \mathrm{~mA} / \mathrm{cm}^{2}$ range. This range was chosen because it yielded more adherent and smoother films compared to higher current ranges, which tended to be less adherent, powdery and brittle. This could possibly be due to the increased Hydrogen evolution and fixation in the metal film during deposition, which results in internal stresses and causes the film to crack [9]. In all cases, however, films with thickness above $150-200 \mathrm{~nm}$ suffered from poor adhesion and often peeled off. Once the electrodeposition was complete, the samples were washed with DI water, blown dry and characterised using Energy Dispersive X-Ray (EDX) analysis and Vibrating Sample Magnetometry (VSM).

3 Compositional analysis A Jeol 6500 EDX system was used to find the composition of the deposited metal film. For each sample, EDX data was acquired at multiple locations, the mean $\mathrm{Ni}$ and $\mathrm{Pd}$ atomic percentages were calculated and were normalised to $100 \%$ by ignoring the EDX peak from the Si substrate. Fig. 2 shows a section of the cyclic voltammetry curves for individual Ni baths and Pd baths of different concentrations.

When a PdNi bath is formed with the concentrations of $\mathrm{NiSO}_{4}$ and $\mathrm{Pd}($ en $) \mathrm{Cl}_{2}$ mentioned in Fig. 2, we get a bath with the the $\mathrm{Ni}$ atomic fractions listed in Table. 2. It is assumed that the electrodeposition reactions are independent and hence the ratio of the currents in the individual baths at the chosen deposition potential determines the composition of the film.

Table. 2 lists the composition of films electrodeposited from the different bath solutions and measured by EDX analysis. The ratio of the currents at the deposition potential were found from the cyclic voltammetry curves in Fig. 2 and compared with the film composition measured by EDX.

Table 2 EDX results for films deposited using solutions of various $\mathrm{Ni}$ atomic percentages and different deposition potentials.

\begin{tabular}{cccc}
\hline Ni\% & Dep. Potential & \multicolumn{2}{c}{ Ni\%(Film) } \\
(solution) & $(\mathrm{V})$ & Nominal & EDX \\
\hline 50.0 & -0.80 & $\approx 0.003$ & 0 \\
80.0 & -1.24 & 49.3 & 48.0 \\
90.0 & -1.60 & 72.1 & 69.2 \\
\hline
\end{tabular}

Table. 2 shows good agreement between the nominal and EDX values of the film composition and shows that the assumption of the currents in the PdNi bath being a sum of the individual $\mathrm{Ni}$ and Pd currents is borne out. The composition of the electrochemical bath does not affect the film composition and it is the ratio of the currents generated by the individual electrochemical reactions at the deposition potential that governs the film composition. 

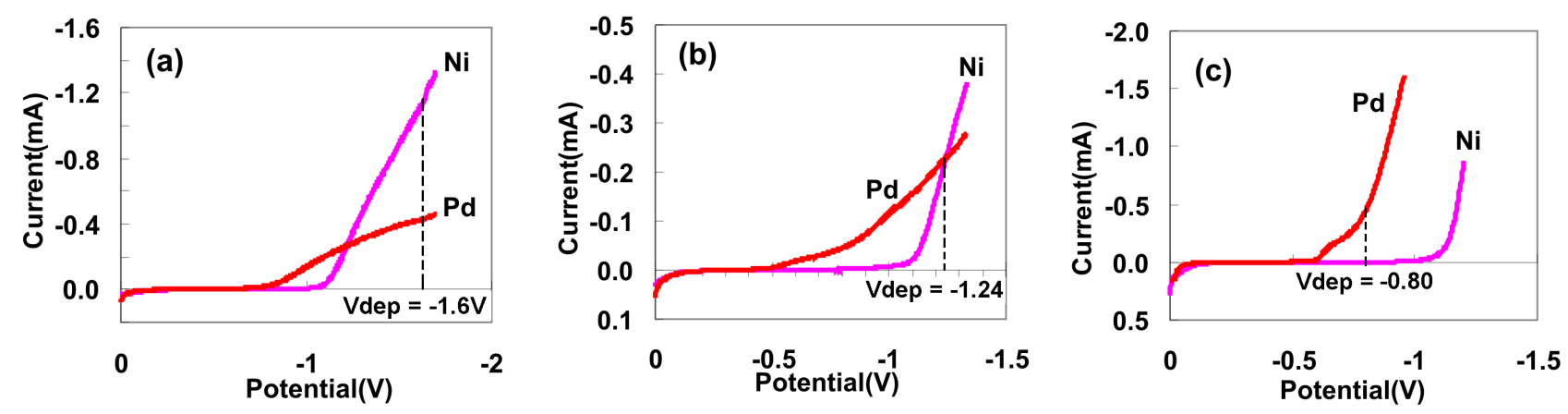

Figure 2 Sections of cyclic voltammetry curves for three different Ni baths and Pd baths with the deposition potentials used for depositing PdNi films from a PdNi bath using the same $\mathrm{NiSO}_{4}$ and $\mathrm{Pd}(\mathrm{en}) \mathrm{Cl}_{2}$ concentrations.(a) $\mathrm{NiSO}_{4}=49.95 \mathrm{~g} / \mathrm{L}, \mathrm{Pd}(\mathrm{en}) \mathrm{Cl}{ }_{2}=$ $5.55 \mathrm{~g} / \mathrm{L}, \mathrm{V}_{\text {dep }}=-1.6 \mathrm{~V}$. (b) $\mathrm{NiSO}_{4}=44.40 \mathrm{~g} / \mathrm{L}, \mathrm{Pd}(\mathrm{en}) \mathrm{Cl}_{2}=11.10 \mathrm{~g} / \mathrm{L}, \mathrm{V}_{\mathrm{dep}}=-1.24 \mathrm{~V}$.(c) $\mathrm{NiSO}_{4}=27.75 \mathrm{~g} / \mathrm{L}, \mathrm{Pd}(\mathrm{en}) \mathrm{Cl}{ }_{2}=27.75 \mathrm{~g} / \mathrm{L}$, $\mathrm{V}_{\text {dep }}=-0.80 \mathrm{~V}$

From Fig. 2, it is seen that solutions with higher $\mathrm{Ni}$ atomic fractions show a crossover point beyond which the magnitude of the Ni current is larger than that of the Pd current. This change in current magnitudes allows deposition of films that are Pd-rich, Ni-rich or have similar Pd and Ni content from the same solution by varying the deposition potential. Solutions with lower Ni atomic fractions do not exhibit this crossover even till a deposition potential of $-3 \mathrm{~V}$ and therefore will deposit only Pd-rich films.

4 Magnetic Characterisation The magnetic characteristics of the deposited films were studied using a Oxford Instruments Aerosonic 3001 VSM. Fig. 3 shows an M-H trace from two PdNi alloy films with different compositions. Fig. 3 shows that the samples exhibit a well defined hysteresis loop and the sample with larger Ni concentration exhibits a higher saturation as well as remnant magnetisation. The table in Fig. 3 inset lists the saturation magnetisation per unit mass $M_{s}$ and remnant magnetisation per unit mass $M_{r}$ for different $\mathrm{Ni}$ atomic fractions. Ferromagnetic behaviour is observed for $\mathrm{Ni}$ fractions above $30 \%$, but may be observed at even lower concentrations [10]. The saturation magnetisation increases with $\mathrm{Ni}$ fraction in the film. The remnant magnetisation also increases with $\mathrm{Ni}$ fraction except for the pure Ni film, in which case a wider hysteresis loop with higher coercivity and lower remnant magnetisation was observed.

5 Device Measurement CNT dispersions were made by mixing $1 \mathrm{mg}$ of dry CNT powder in 1,2-dichloro benzene followed by sonication for 45 minutes. The dispersion was spun onto an n-type $<100>$ Si wafer with $20 \mathrm{~nm}$ thermal oxide and ohmic Al back contact. The wafer was covered with photoresist and patterns were defined using an EVG620 mask aligner. The thermal oxide in the windows was etched using 20:1 HF followed by rinsing with DI water. Electrodeposition was then performed us-

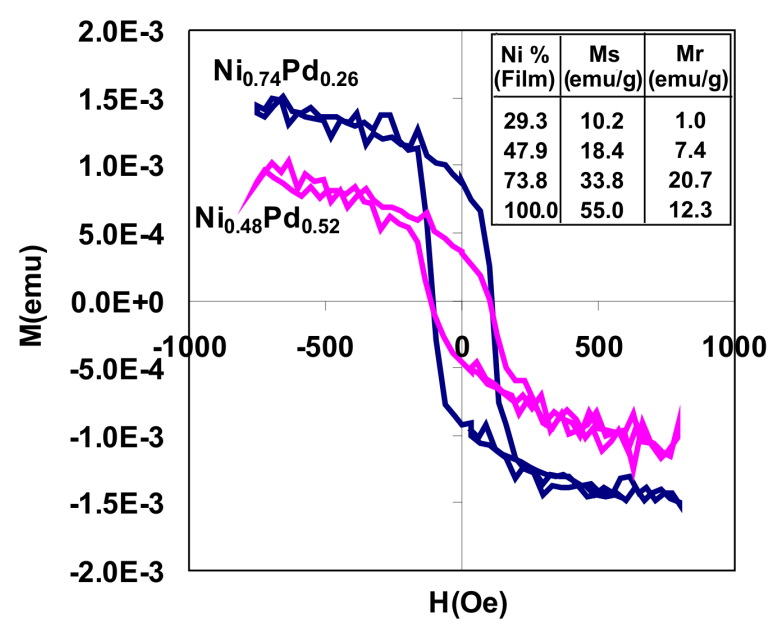

Figure $3 \mathrm{M}-\mathrm{H}$ traces for $\mathrm{Ni}_{0.74} \mathrm{Pd}_{0.26}$ and $\mathrm{Ni}_{0.48} \mathrm{Pd}_{0.52}$ alloy compositions. The table in the inset shows Saturation magnetisation $\mathrm{M}_{\mathrm{s}}$ and Remnant magnetisation $\mathrm{M}_{\mathrm{r}}$ values for different $\mathrm{Ni}$ content in deposited films.

ing the PdNi bath listed in Table. 1 and the devices were electrically characterised using a DC prober and Agilent 4155C semiconductor parameter analyser. An SEM image of a CNT contacted by two PdNi pads is shown in Fig. 4. The CNT is about $4 \mu \mathrm{m}$ long and the two ends are embedded in the PdNi contacts. Other nanotubes are also seen on the substrate and though no other CNT is visibly contacted by both PdNi pads, there are a few which cross the contacted CNT. The implications of such crossings and possible connections are yet to be considered. For electrical characterisation, the device was biased as shown in Fig. 1 to ensure that the two PdNi-Si Schottky barriers are always reverse biased. Fig. 5 shows the variation of the 


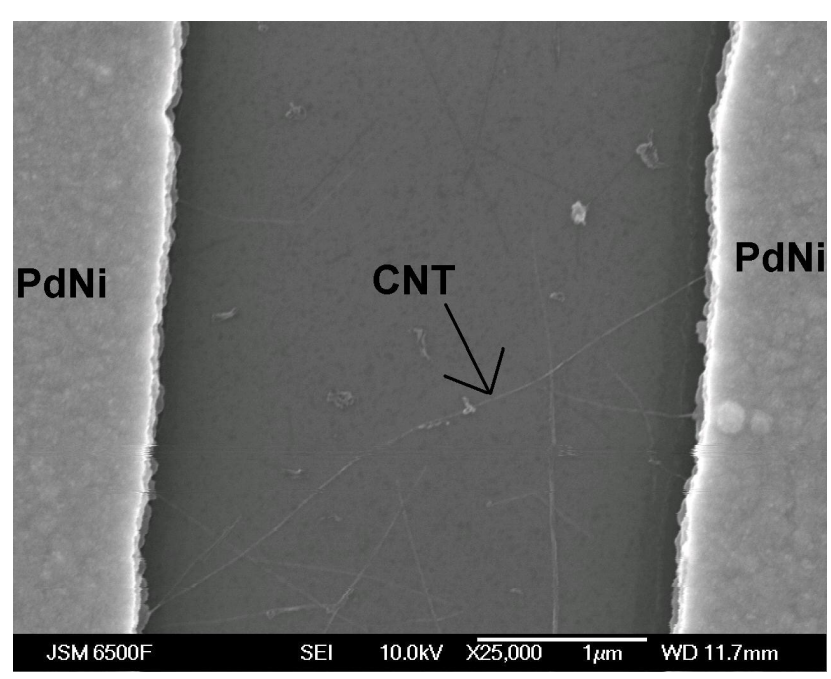

Figure $4 \mathrm{CNT}$ contacted by electrodeposited PdNi

drain, source and gate currents with the gate voltage, while the inset of Fig. 5 shows the variation of drain current with drain to source potential. The resistance of the device could also be used to investigate the presence of a CNT since devices with CNTs absent were observed to have larger resistances ( $>\mathrm{G} \Omega$ ) compared to devices with CNTs $(\mathrm{M} \Omega)$.

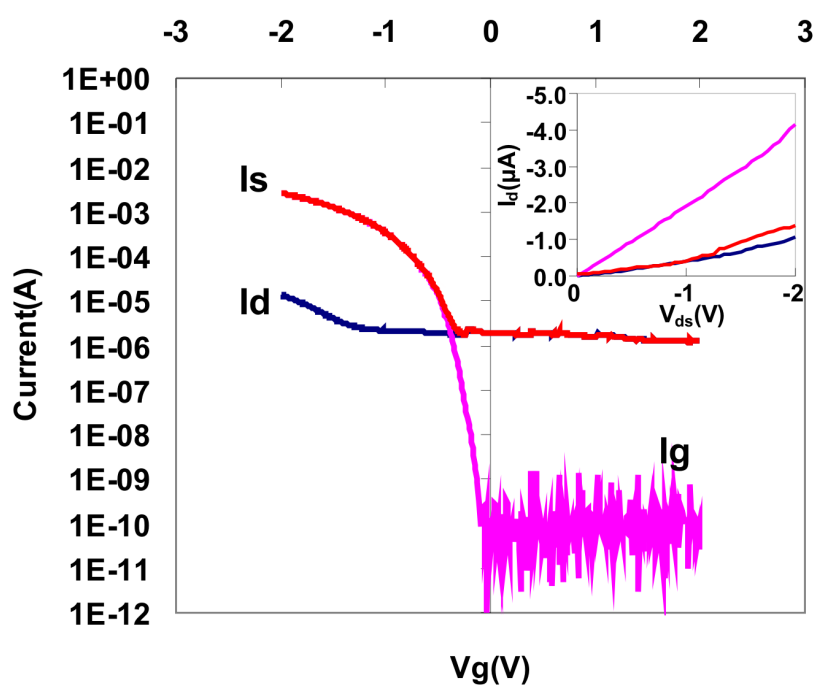

Figure 5 Variation of drain, source and gate currents with gate voltage for CNT device with $\mathrm{Ni}_{0.40} \mathrm{Pd}_{0.60}$ contacts. For this measurement, $\mathrm{V}_{\mathrm{d}}=-2 \mathrm{~V}, \mathrm{~V}_{\mathrm{s}}=0 \mathrm{~V}$ and $\mathrm{V}_{\mathrm{g}}$ was swept from $-2 \mathrm{~V}$ to $2 \mathrm{~V}$. The inset shows the dependence of $\mathrm{I}_{\mathrm{ds}}$ when $\mathrm{V}_{\mathrm{g}}=0 \mathrm{~V}$, $\mathrm{V}_{\mathrm{s}}=-1 \mathrm{~V}$ and $\mathrm{V}_{\mathrm{ds}}$ is swept from $-1 \mathrm{~V}$ to $-3 \mathrm{~V}$

Fig. 5 proves that the device architecture successfully channels most of the current through the CNT when the
PdNi-Si Schottky barriers are reverse biased. In the negative $\mathrm{V}_{\mathrm{gs}}$ region the source-gate Schottky barrier is forward biased, resulting in large values of $I_{g}$ and $I_{s}$. As the gate voltage goes positive, the source-gate Schottky barrier switches off and the gate leakage current $\left(\mathrm{I}_{\mathrm{g}}\right)$ reduces to a few nanoamperes. The source current however, stabilises at the same level as the drain current, indicating that all current in the device is between the source and the drain. The CNT connected between these two will therefore conduct all of this current. The gate current in Fig. 5 shows the excellent Schottky barrier formed between PdNi and $\mathrm{Si}$. The on current is higher than the off-current by 7 orders of magnitude and the barrier height is $0.80 \mathrm{eV}$.

A small increase of an order of 10 in drain current for low gate voltages is observed and may be due to transistor operation, but further measurements are required to support this assumption. The next step in this study is to examine the behaviour of the device in magnetic fields.

6 Conclusion The structure of a three terminal spintronic CNT device with electrodeposited PdNi contacts was presented. Electrodeposition of the PdNi films and their electrical and magnetic characterisation was described. The CNT device fabrication process and results from initial electrical characterisation confirm that the PdNi contacts channel most of the current through the CNT when the PdNi-Si Schottky barriers are reverse biased.

Acknowledgements We thank Dr. P. A. J de Groot and Daowei Wang for their assistance in using the VSM. We also thank Dr. Shuncai Wang and Dr. David Claudio Gonzalez for their assistance with EDX measurements.

\section{References}

[1] P. Avouris, Accounts of Chemical Research 35(12), 10261034 (2002).

[2] P. Avouris, Z. Chen, and V. Perebeinos, Nat Nano 2(10), 605-615 (2007).

[3] A. Javey, J. Guo, Q. Wang, M. Lundstrom, and H. Dai, Nature 424(6949), 654-657 (2003).

[4] V. Derycke, R. Martel, J. Appenzeller, and P. Avouris, Nano Letters 1(9), 453-456 (2001).

[5] S. Sahoo, T. Kontos, J. Furer, C. Hoffmann, M. Graber, A. Cottet, and C. Schonenberger, Nat Phys 1(2), 99-102 (2005).

[6] N. Tombros, S. J. van der Molen, and B. J. van Wees, Physical Review B 73(23), 233403 (2006).

[7] M. E. Kiziroglou, A. A. Zhukov, M. Abdelsalam, X. Li, P. A. J. de Groot, P. N. Bartlett, and C. H. de Groot, IEEE Transactions on Magnetics 41(10), 2639-2641 (2005).

[8] S. E. Nam and K. H. Lee, Journal of Membrane Science 170(1), 91-99 (2000).

[9] M. Schlesinger and M. Paunovic, Modern Electroplating, fourth edition (John Wiley and Sons, 2000).

[10] J. Crangle and W. R. Scott, Journal of Applied Physics 36(3), 921-928 (1965). 